\title{
1D Nanowires of 2D Layered Materials: A New Frontier in Nanomaterials
}

\author{
Eli Sutter, Peter Sutter \\ University of Nebraska-Lincoln, Lincoln, United States of America; \\ esutter@unl.edu
}

Semiconductor nanowires have mostly been synthesized from conventional three dimensional (3D) crystalline materials. Layered crystals, in which covalently bonded sheets are held together by weaker van der Waals forces, have emerged as a class of materials with extraordinary properties not found in 3D crystals. Shaping layered materials into nanowires could open up new, tunable structural, optoelectronic, and electronic transport/device characteristics.

Here, we discuss the realization of this vision, namely the synthesis and emerging properties of van der Waals nanowires of layered crystals, formed by combining the concepts of vapor-liquid-solid (VLS) growth and van der Waals epitaxy. We demonstrate the possibility of forming nanowires of germanium (II) sulfide (GeS), a 2D/layered chalcogenide semiconductorwith anisotropic structure [1], by a VLS process [2]. High-quality van der Waals nanowires crystallize with layering along the wire axis and show bright, size dependent band-edge luminescence [3], [4]. A strong propensity for forming screw dislocations, often found for layered crystals [4], introduces extraordinary properties without analogues in 3D-crystalline nanowires. Eshelby twist, induced by a torque on the ends of a cylindrical solid due to the stress field of an axial dislocation, causes a chiral structure of the layered nanowires and leads to spontaneous, size-tunable twist moiré patterns between the van der Waals layers along the wires. Using tailored growth protocols complex structures can be obtained that are impossible to realize in planar van der Waals stacks, including homojunctions between twisted (dislocated) and ordinary layered (dislocation-free) segments as well as continuously variable Eshelby twist translating into a seamless progression of helical moiré patterns [5]. Combined electron diffraction and local (nanometer-scale) optoelectronic measurements using cathodoluminescence and electron-energy loss spectroscopy show the correlation between the interlayer twist and locally excited light emission/optical absorption that is due to progressive changes in the lattice orientation and in the interlayer moiré registry along the nanowires. These findings demonstrate an avenue for the scalable fabrication of van der Waals structures with defined twist angles for the emerging field of twistronics, in which interlayer moiré patterns are realized along a helical path on a nanowire instead of a planar interface.

[1] E. Sutter, B. Zhang, M. Sun, P. Sutter, ACS Nano 13, 9352 (2019).

[2] E. Sutter, P. Sutter, ACS Applied Nano Materials 1, 1042 (2018).

[3] P. Sutter, C. Argyropoulos, E. Sutter, Nano Letters 18, 4576 (2018).

[4] P. Sutter, S. Wimer, E. Sutter, Nature 570, 354 (2019).

[5] P. Sutter, J.-C. Idrobo, and E. Sutter, Adv. Funct. Mater. 31, 2006412 (2021).

Keywords: layered 2D materials, nanowires, GeS, cathodoluminescence, nanobeam electron diffraction 